\title{
Extra-Articular Ganglion Cysts around the Knee Joint
}

\author{
Sang-Eun Park, MD, Karnav Panchal, MD, Jae-Jung Jeong, MD, Young-Yul Kim, MD, Jong-Hun Ji, MD, \\ Sung-Ryeoll Park, MD, and Min-Kyu Park, MD \\ Department of Orthopedic Surgery, Daejeon St. Mary Hospital, The Catholic University of Korea College of Medicine, Daejeon, Korea
}

Purpose: The purpose of this study was to report clinical results of open excision of extra-articular ganglion cysts around the knee joint combined with arthroscopic management of intra-articular pathologies if present.

Materials and Methods: Of the total 107 cases of cystic lesions around the knee, 23 cases of extra-articular ganglion cysts were reviewed between January 2006 and July 2011. There were 13 males and 10 females with a mean age of 48 years (range, 30 to 73 years). The mean follow-up duration was 40 months (range, 30 to 60 months). Preoperative magnetic resonance imaging (MRI) scan was done in all cases. Open surgical excision of the cyst was performed after arthroscopic management of intra-articular pathologies in all but 1 case. At the last follow-up, Lysholm and International Knee Documentation Committee (IKDC) scores were evaluated and MRI was conducted to detect recurrence.

Results: The mean Lysholm and IKDC scores showed significant improvement ( $\mathrm{p}=0.005$ and 0.013 , respectively).The location of the cysts was anterior in 9, lateral in 7, medial in 6, and posterosuperior in 1. Intra-articular pathologies were found in 16/23 cases (69.6\%). In 10/23 cases (43\%), the cyst was connected to the knee joint. Three months postoperative MRI did not show any recurrence of ganglion cysts except for 1 case.

Conclusions: In the treatment of extra-articular ganglion cysts, MRI can be useful for detecting intra-articular lesions and connecting orifices, and arthroscopic management of intra-articular pathologies with open excision of the cyst should be considered as a viable treatment option.

Keywords: Knee, Popliteal ganglion cyst, Intra-articular, Magnetic resonance imaging, Combined excision

\section{Introduction}

In general, ganglion cysts around the knee joint are classified into intra-articular, extra-articular soft tissue, periosteal, and intra-osseous ganglion cysts. Several studies have described intraarticular ganglion cysts regarding their locations and shapes in detail; however, extra-articular soft tissue ganglion cysts have been reported in only a few articles ${ }^{1-4)}$. Extra-articular ganglion cysts usually appear as uni- or multi-septate cysts on magnetic

Received May 8, 2015; Revised June 22, 2015;

Accepted June 23, 2015

Correspondence to: Jae-Jung Jeong, MD

Department of Orthopedic Surgery, Daejeon St. Mary Hospital, The Catholic University of Korea College of Medicine, 64 Daeheung-ro, Jung-gu, Daejeon 34943, Korea

Tel: +82-42-220-9530, Fax: +82-42-221-0429

E-mail: jjeong@catholic.ac.kr

This is an Open Access article distributed under the terms of the Creative Commons Attribution Non-Commercial License (http://creativecommons.org/licenses/by-nc/4.0/) which permits unrestricted non-commercial use, distribution, and reproduction in any medium, provided the original work is properly cited. resonance imaging (MRI). Thus, they have been just cited as differential diagnosis of cystic lesions around the knee joint such as meniscal cysts and synovial cysts or are mostly reported with associated findings such as peroneal nerve palsy and bony remodeling ${ }^{5-11)}$.

Among these cysts, Baker's cysts are the most frequently encountered cystic masses around the knee. They often have an intra-articular communication with associated intra-articular pathologies. Bickel et al. ${ }^{12}$ reported the incidence of intra-articular communication in Baker's cysts as 54/83 cases (65\%). MRI studies of popliteal cysts demonstrated the presence of an association with one or more intra-articular lesions in $87 \%-98 \%$ of cases $^{13,14)}$. Extra-articular ganglion cysts, other than Baker's cysts, are relatively uncommon, and clinical studies on these cysts are rare. Furthermore, few literatures have documented on extra-articular ganglion cysts around the knee communicating with the joint.

The purpose of this study was to report clinical results of open excision of extra-articular ganglion cysts around the knee joint combined with arthroscopic evaluation and management of intra-articular pathologies if present and to investigate the inci- 
dence of communication between these extra-articular ganglion cysts and the knee joint.

\section{Materials and Methods}

Institutional Review Board approval was obtained before patients were enrolled in the study. From January 2006 to June 2011, we treated a total of 107 cases of cystic lesions around the knee joint. Of these, we excluded 84 cases with a Baker's cyst (78 cases), a cruciate ligament ganglion cyst (4 cases), and a meniscal cyst ( 2 cases). The remaining 23 cases with symptomatic extra- articular ganglion cysts were retrospectively reviewed. In all cases, preoperative MRI evaluation was conducted to confirm the presence of an extra-articular ganglion cyst and to investigate any associated intra-articular pathologies and intra-articular connection of these extra-articular cysts. Among the 23 cases, 2 cases with a previous history of surgical excision of the cyst presented with a recurrence of the cyst. They were referred to our hospital after primary surgical excision that had been performed without preoperative MRI evaluation. Our surgical indications included a symptomatic knee joint mass with pain or discomfort and symptoms refractory to 3 to 6 months of conservative treatment in the

Table 1. Details of Extra-Articular Ganglion Cysts

\begin{tabular}{|c|c|c|c|c|c|}
\hline No. & Age (yr) & Sex & Location $^{\text {a) }}$ & Size $(\mathrm{cm})$ & Comment \\
\hline 1 & 54 & $\mathrm{~F}$ & Medail-anterosuperior & $1.0 \times 1.2 \times 0.9$ & MFC: chondromalacia (Outerbridge grade I) \\
\hline 2 & 65 & $\mathrm{~F}$ & Lateral-posteroinferior & $1.9 \times 2.1 \times 0.6$ & Proximal tibia: chondromalacia \\
\hline 3 & 44 & M & Anterior-superomedial & $\begin{array}{l}\text { I/A size, } 1.3 \times 2.0 \times 0.9 \\
\text { E/A size, } 2.1 \times 1.6 \times 2.0\end{array}$ & Intra-articular ganglion extending to extra-articular side \\
\hline 4 & 62 & $\mathrm{~F}$ & Anterior-superolateral & $\begin{array}{l}\text { I/A size, } 3.2 \times 2.0 \times 1.6 \\
\text { E/A size, } 1.1 \times 1.6 \times 1.1\end{array}$ & $\begin{array}{l}\text { MFC: Outerbridge grade II, focal ACD }(3 \times 3 \mathrm{~mm} \text { sized }) \\
\text { Intra-articular ganglion extending to extra-articular side }\end{array}$ \\
\hline 5 & 53 & M & Anterior-inferomedial & $1.0 \times 1.1 \times 1.4$ & \\
\hline 6 & 33 & M & Medial-anterosuperior & $2.1 \times 1.2 \times 1.1$ & \\
\hline 7 & 30 & $\mathrm{~F}$ & Lateral-posteroinferior & $3.0 \times 3.0 \times 2.0$ & Proximal tibia: condromalacia \\
\hline 8 & 37 & M & Medial-posterosuperior & $1.5 \times 1.0 \times 0.8$ & Patella (medial facet): chondromalacia (Outerbridge grade I-II ) \\
\hline 9 & 48 & $\mathrm{~F}$ & Medial-anterosuperior & $\begin{array}{l}\text { I/A size, } 2.3 \times 0.7 \times 0.8 \\
\text { E/A size, } 3.0 \times 3.0 \times 2.0\end{array}$ & Intra-articular ganglion extending to extra-articular side \\
\hline 10 & 44 & M & Medial-posterosuperior & $6.1 \times 3.8 \times 2.1$ & \\
\hline 11 & 44 & M & Medail-anterosuperior & $1.1 \times 3.3 \times 1.1$ & Meidal meniscus tear \\
\hline 12 & 38 & M & Anterior-superolateral & $4.0 \times 7.0 \times 4.0$ & Intramuscular: gastrocnemius muscle \\
\hline 13 & 34 & M & Medial-posterosuperior & $2.5 \times 2.1 \times 0.6$ & Multilobulated cystic nodules \\
\hline 14 & 38 & M & Anterior-superolateral & $3.3 \times 1.2 \times 1.9$ & \\
\hline 15 & 71 & $\mathrm{~F}$ & Anterior-inferomedial & $1.0 \times 0.6 \times 0.6$ & Medial meniscus tear \\
\hline 16 & 41 & M & Lateral-posteroinferior & $2.6 \times 2.6 \times 4.9$ & Multiseptated mass in the proximal portion of peroneus muscle \\
\hline 17 & 42 & $\mathrm{~F}$ & Lateral-anteroinferior & $1.2 \times 1.8 \times 1.8$ & Cystic lesion adjacent to the iliotibial band \\
\hline 18 & 38 & M & Anterior-inferomedial & $1.1 \times 2.0 \times 0.8$ & \\
\hline 19 & 44 & M & Lateral-anterosuperior & $3.5 \times 3.8 \times 1.8$ & $\begin{array}{l}\text { MFC: ACD } \\
\text { Medial meniscus tear }\end{array}$ \\
\hline 20 & 67 & M & Anterior-inferomedial & $3.6 \times 0.6 \times 0.9$ & $\begin{array}{l}\text { Medial meniscus tear } \\
\text { Multiseptated ganglion cyst }\end{array}$ \\
\hline 21 & 73 & M & Anterior-inferomedial & $0.8 \times 1.6 \times 0.3$ & Medial meniscus tear \\
\hline 22 & 44 & M & Lateral-anterosuperior & $2.3 \times 1.9 \times 1.2$ & Proximal tibia: chondromalacia \\
\hline 23 & 55 & $\mathrm{~F}$ & Lateral-anteroinferior & $3.0 \times 1.2 \times 1.3$ & $\begin{array}{l}\text { MFC: ACD } \\
\text { Proximal tibia: chondromalacia }\end{array}$ \\
\hline
\end{tabular}

MFC: medial femoral condyle, I/A: intra-articular, E/A: extra-articular, ACD: articular cartilage defect.

${ }^{a}$ Superior or inferior location of the cyst in reference to the joint line. Cyst size was measured by the longest line of the cyst on each coronal, sagittal and axial magnetic resonance imaging scan. 
form of physical therapy or medication, and preoperative MRI evidence of the cyst.

There were 13 males and 10 females and their mean age was 48 years (range, 30 to 73 years). The mean follow-up duration was 40 months (range, 30 to 60 months). The mean body mass index (BMI) was 22.5 (range, 19.1 to 26.8). The details of the extra-articular ganglion cysts are presented in Table 1 . The cystic lesions showed low signal intensity on T1-weighted images and high signal intensity on T2-weighted images. For any extra-articular cysts with MRI-evidence of intra-articular connection or combined intra-articular pathologies, arthroscopic evaluation and management of the intra-articular pathologies and intra-articular cyst decompression were followed by open surgical excision of the extra-articular ganglion cyst and ligation of the connecting channel. In one case where MRI did not show any intra-articular pathology or communication of the extra-articular cyst with the knee joint, only open surgical excision of the extra-articular cyst was performed; however, we took care to ensure there was no communicating stalk or orifice of the cyst within the knee joint during surgical excision.

\section{Surgical Procedure}

Arthroscopic evaluation was performed in all cases except for 1 case without any associated intra-articular pathology or intra-articular communication of the cyst. During arthroscopic examination, after confirming the presence of intra-articular pathologies, we attempted to check for communication of the extra-articular cyst with the knee joint. Once an intra-articular cystic lesion was observed around the infra-patellar fat pad, an arthroscopic shaver was used to clear the fat pad and to decompress the intraarticular cystic lesions, and then the dissected synovium was repaired using absorbable sutures (2.0 prolene) (Fig. 1). If arthroscopy showed associated intra-articular lesions such as articular cartilage defects (grade III-IV) and meniscal tears, we performed debridement of the articular cartilage defect or partial meniscectomy. After confirming the presence of an intra-articular connecting orifice on arthroscopy, we dissected extra-articular ganglion cysts carefully and searched for the stalk of the ganglion cyst communicating to the knee joint. Complete excision of the ganglion cyst and ligation of the stalk was performed using silk no. 3-0 (Fig. 2). For two postoperative weeks, elastic bandage compression dressing was applied. Ambulation was allowed at postoperative week 1 or 2 after confirming the patient found
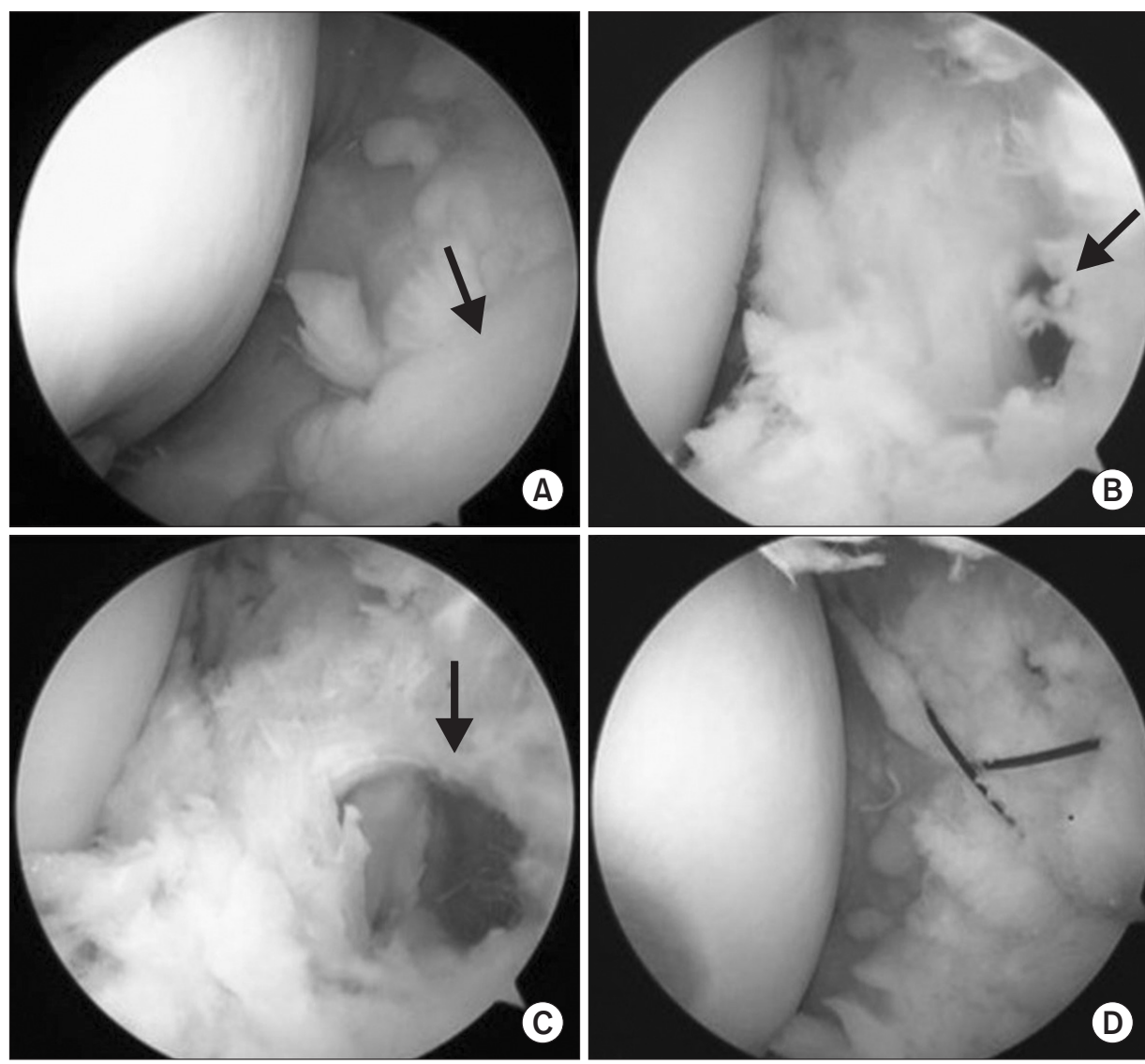

Fig. 1. Arthroscopic findings. (A) Intraarticular cyst seen from the joint with synovial bulging. (B, C) Arthroscopic cyst decompression showing yellowish fluid coming out from the cyst and the connecting orifice of the cyst. (D) Repair of the capsule and synovium using absorbable sutures (2.0 prolene). 

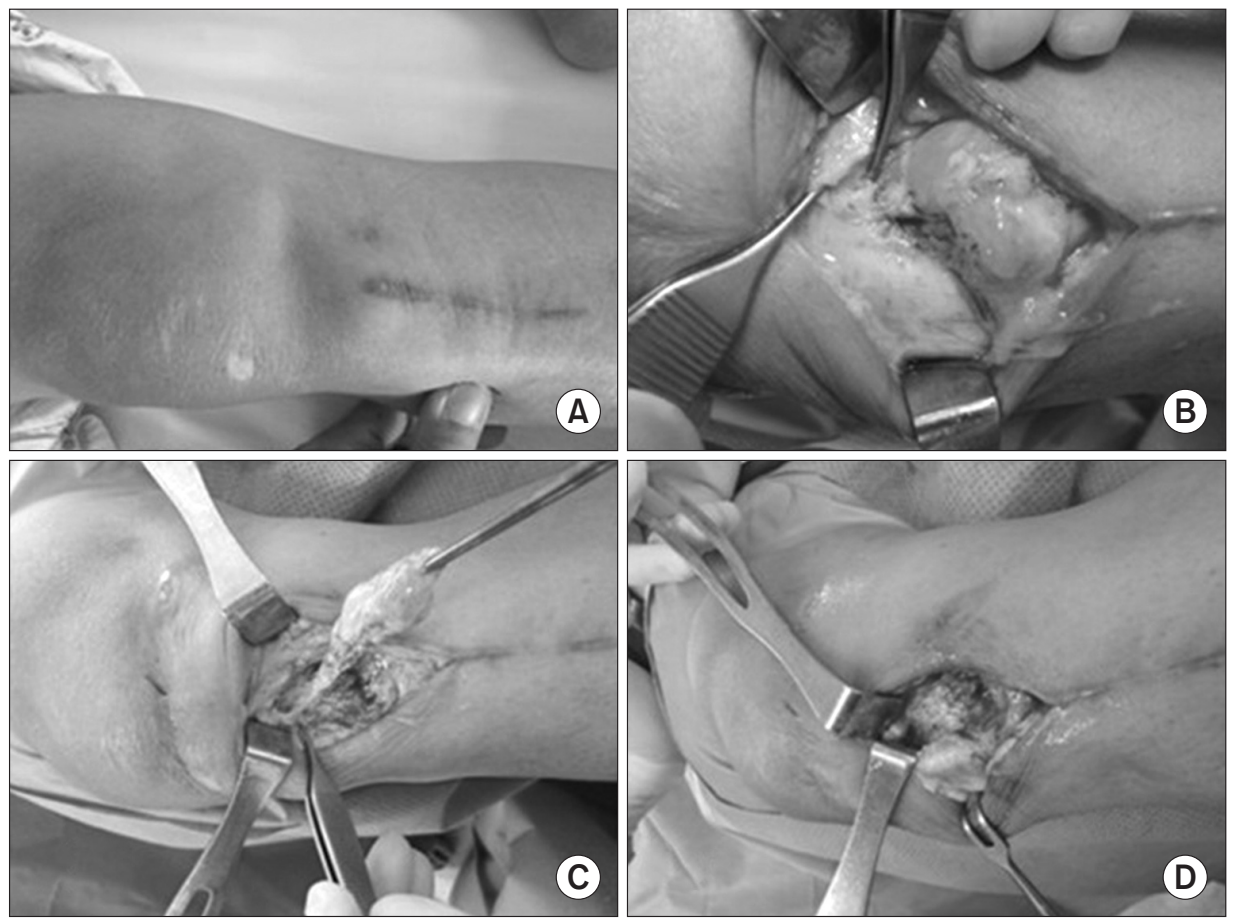

Fig. 2. Open surgical excision of a recurrent extra-articular ganglion cyst. (A) The recurrent mass was located on the proximal aspect of the knee joint. (B-D) The large ganglion sac $(10 \times 5 \times 4 \mathrm{~cm})$ was found connected to the knee joint through the patellar tendon and fat pad and was removed completely. walking tolerable. Depending on adhesion of the extra-articular cystic wall to the joint capsule, the period of immobilization was adjusted. If a medially located cyst was attached firmly to the medial joint capsule or the medial collateral ligament, complete removal of the cyst resulted in partial removal of the joint capsule, so these patients were immobilized for 2 weeks to promote soft tissue and joint capsule healing and prevent further damage to the repaired capsule. Follow-up MRI was performed 3 months postoperatively to detect recurrence and to assess the healing status of the repaired capsule or synovium.

\section{Results}

The locations of the extra-articular cysts around the knee are listed in Table 2.

One cyst was found in the posterosuperior aspect of the knee joint. Medially located cysts were located adjacent to the joint line, the medial collateral ligament, or the posterior border of the vastus medialis muscle. Cysts on the lateral aspect were located adjacent to the joint line, the fibular head, an intramuscular location (gastrocnemius muscle), the proximal portion of the peroneus muscle, or adjacent to the iliotibial band.

On MRI, the size of the cyst in longitudinal diameter ranged from 1.0 to $7.0 \mathrm{~cm}(2.7 \pm 1.6 \mathrm{~cm})$ and in transverse diameter from 0.6 to $4.0 \mathrm{~cm}(2.0 \pm 1.0 \mathrm{~cm})$. Lysholm and International Knee Documentation Committee scores were significantly improved
Table 2. Location of Extra-Articular Ganglion Cysts around the Knee Joint

\begin{tabular}{ll}
\hline \multicolumn{1}{c}{ Location $^{\text {a) }}$} & Cases \\
\hline Anterior aspect of the knee & 3 \\
Superolateral & 1 \\
Superomedial & 5 \\
Inferomedial & \\
Lateral aspect of the knee & 3 \\
Anterorsuperior & 1 \\
Anteroinferior & 3 \\
Posteroinferior & \\
Medial aspect of the knee & \\
Anterosuperior & 4 \\
Posteroinferior & 2
\end{tabular}

${ }^{a)}$ Superior or inferior position of the cyst within the knee joint is in reference to the level of the joint line. Anterior or posterior position of the cyst within the knee joint is in reference to the medial and lateral collateral ligament.

from a preoperative mean of $69 \pm 15$ (range, 35 to 86 ) and $55 \pm 20$ (range, 17 to 83 ), respectively, to a postoperative mean of $95 \pm 8$ (range, 78 to 100 ) and $88 \pm 9$ (range, 56 to 100), respectively ( $\mathrm{p}=0.005$ and 0.013 , respectively). Combined intra-articular pathologies observed in 16/23 cases (69.6\%) of the cases were as follows: articular cartilage defects of the medial femoral condyle or patella (Outerbridge grade III-IV) in 5 cases, chondromalacia 
(Outerbridge grade I-II) in 4 cases, medial meniscus tears in 4 cases, and intra-articular cysts in 3 cases. On MRI evaluation, the extra-articular ganglion cyst was found to be connected to the knee joint through a communicating orifice in 10 cases $(10 / 23$, $43 \%)$. On arthroscopic examination, the communicating orifice was located in the infra-patellar fat pad in 3 cases, in the posterolateral aspect of the knee joint in 1 case (Fig. 3), in the posteromedial aspect in 1 case, and in the superolateral aspect in 1 case (Fig. $4 \mathrm{~B}, \mathrm{C})$. Postoperative MRI at 3 months after surgery did not show any recurrence of these extra-articular ganglion cysts except for 1 case in which we found 2-3 small cysts on MRI in the lateral aspect of the knee joint, but the cysts were very small and the patient was symptom-free.

We sent the excised specimen of the cysts for histopathological examination and confirmed the presence of extra-articular soft tissue ganglion cysts in all cases. On gross examination, all specimens showed cystic mass and on histology, the findings were consistent with ganglion with myxoid change (Fig. 5).

\section{Cases with Recurrence after Primary Surgical Excision}

There was a recurrence in 2 cases after primary surgical excision, in which MRI evaluation was not done prior to excision.
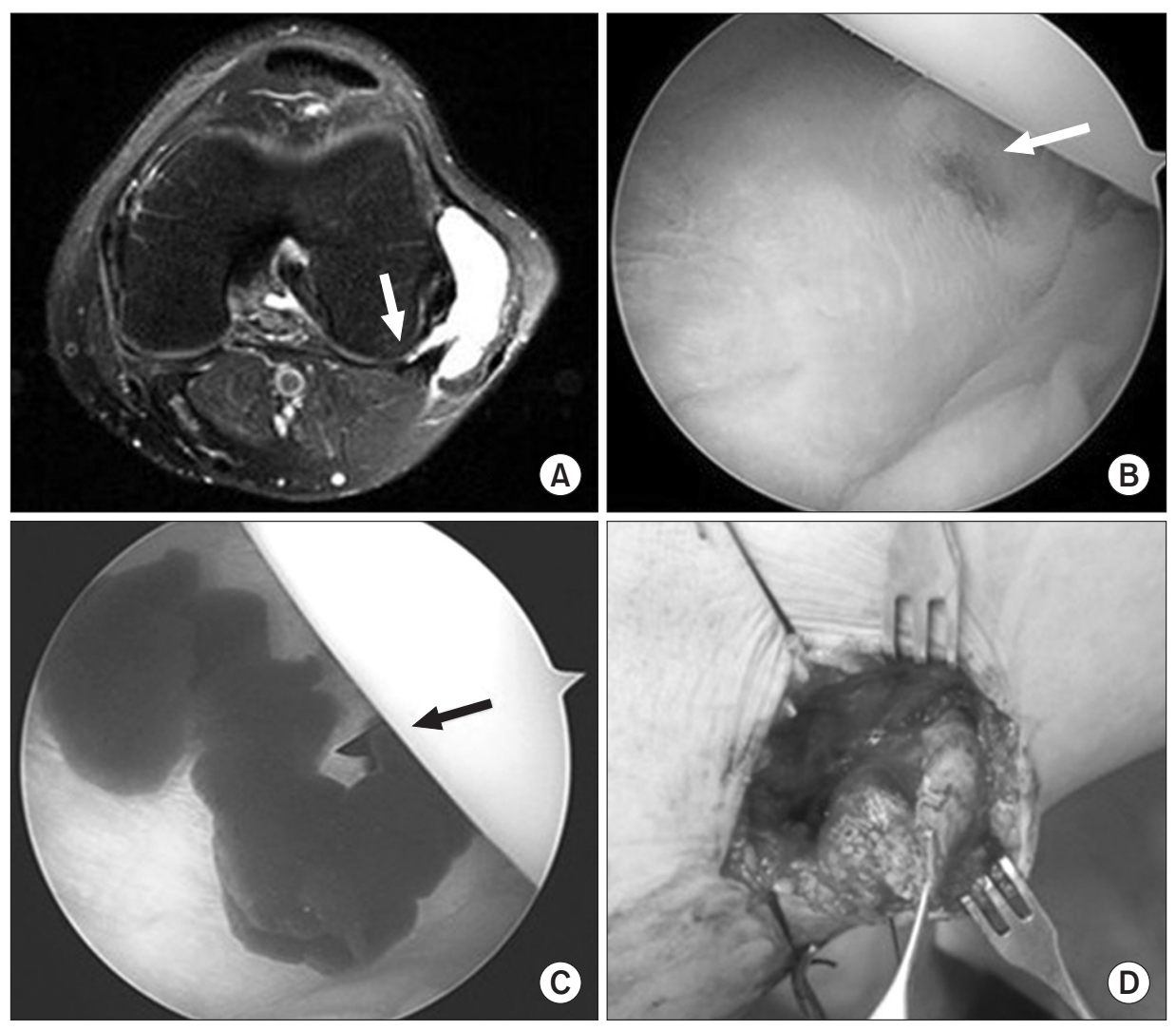

(D)
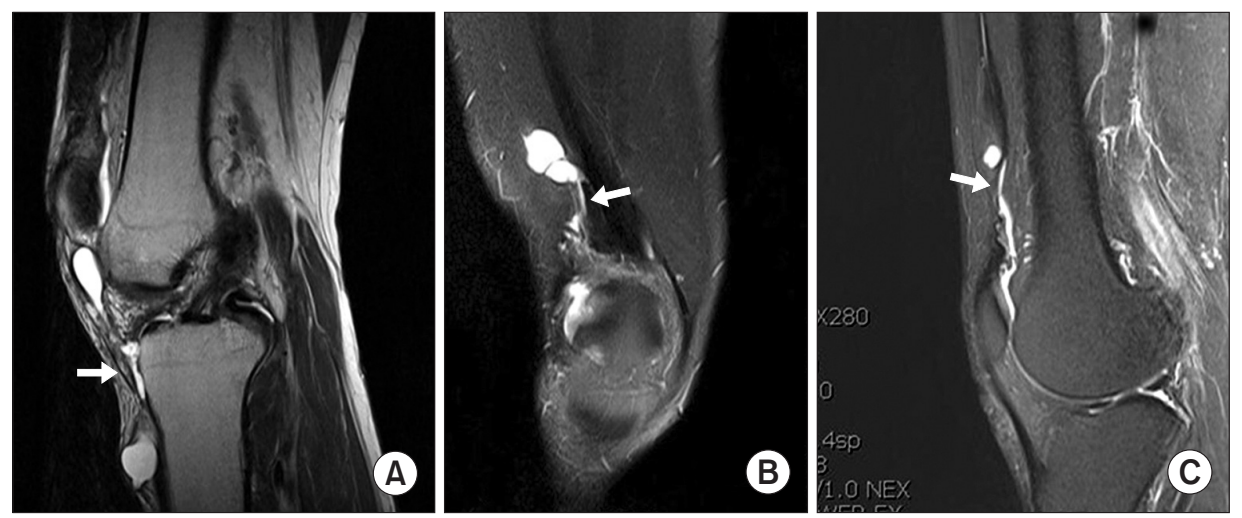

Fig. 4. (A) Intra-articular ganglion extending around the proximal tibia through a communicating stalk (arrow) in the infrapatellar fat pad. (B, C) Magnetic resonance imaging findings of a superolateral extra-articular ganglion. Approximately $3.3 \times 1.2 \times 1.9 \mathrm{~cm}$ in size, well-defined, septate cyst with low T1 and high T2 signal intensity was seen in the vastus lateralis muscle area and the distal femoral level, and this ganglion was connected from the vastus lateralis to the superolateral aspect of the patella. 

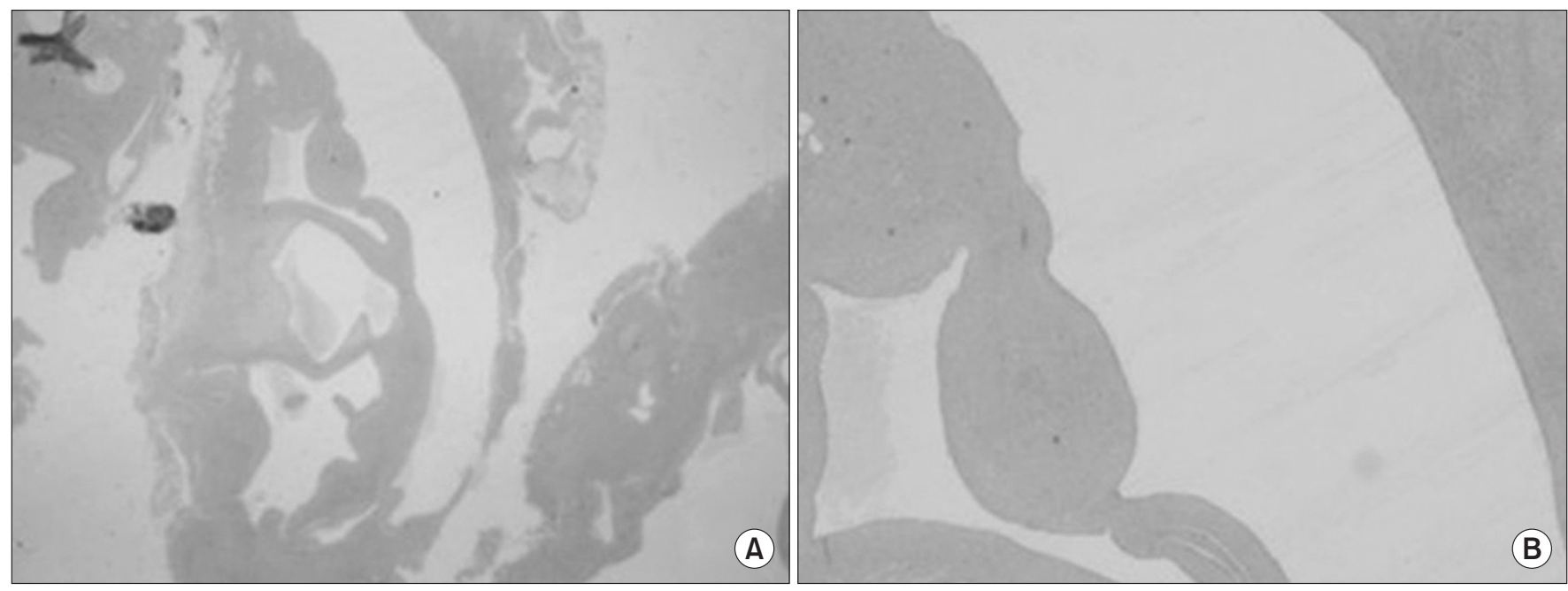

Fig. 5. Histological findings of the cyst consistent with a ganglion with myxoid degeneration $(\mathrm{H} \& \mathrm{E}, \times 10)$. (A) Multiple cystic chambers containing myxoid material. (B) The walls of the cyst composed of fibrous tissue with no specialized lining.

These patients were referred to our clinic from other hospitals. They had a previous history of symptomatic cystic mass in the anteromedial aspect of the proximal tibia. The size of these masses increased slowly after primary excision and the patient started complaining of pain after 2-3 months of surgical procedure without any history of injury or trauma. These patients did not have any underlying disease and preoperative knee range of motion was within normal limits. MRI was performed for evaluation of any intra-articular pathologies and communicating orifice prior to a 2nd operation. The MRI findings showed multiple ganglion cysts extending from medial infrapatellar fat pad to medial prepatellar soft tissue. Low signal intensity on T1-weighted images and high signal intensity on T2-weighted images of the ganglion cysts were found in the medial infrapatellar fat pad (medial patellofemoral joint area). On MRI, a tubular thin tract was suspected and a cystic lesion was observed under pretibial soft tissue at 5 $\mathrm{cm}$ below the joint line. These patients were operated using the same surgical technique as mentioned above (Fig. 2).

In all 3 patients with a recurrence, intra-articular cysts were found extending to the extra-articular ganglion (Fig. 4). In these cases, cystic lesions were found to be connected from the infrapatellar fat pad to the extra-articular aspect around the proximal tibia through a communicating orifice. These patients were also operated using the same surgical technique as mentioned above (Table 1).

\section{Discussion}

The most important finding of our study was that intra-artic- ular pathologies were common in patients with extra-articular ganglion cysts (16/23 cases, $69.6 \%)$ and the extra-articular ganglion cysts were connected to the knee joint through a communicating orifice in 10/23 cases (43\%). In 3 cases (13\%), the cystic lesions were extending from the intra-articular infrapatellar fat pad region to the extra-articular region around the proximal tibia.

A ganglion cyst is a benign cystic mass containing clear, highly viscous fluid that is rich in hyaluronic acid and other mucopolysaccharides within a dense fibrous connective tissue wall without a synovial lining. Cystic lesions around the knee may present with pain, swelling, and discomfort around the knee. MRI of the knee joint helps to confirm the diagnosis of cystic lesions around the knee joint ${ }^{15,16)}$. Extra-articular ganglia may be located in any of the extra-articular soft tissues around the knee, including the joint capsule, tendons, ligaments, busae, muscles, and nerves ${ }^{17-199}$. They rarely communicate with the knee joint ${ }^{13)}$. Albeit rare, the detection of a communication with the joint capsule is very important in planning surgery as a failure to remove the capsular components of these cysts may lead to a recurrence $\mathrm{e}^{15-19)}$.

On MRI evaluation of these cysts in our study, 10/23 cases (43\%) were found to be connected to the knee joint through a communicating orifice that was present in the fat pad, posterolateral or posteromedial aspect of the knee joint, or in the quadriceps tendon. The incidence was lower than that in the study by Kim et al. ${ }^{15)}$ who retrospectively reviewed 30 cases of surgically proven extra-articular soft tissue ganglion cysts around the knee and found a communicating channel between the cyst and the joint in 20 cases (66.7\%). Bickel et al. ${ }^{12)}$ reported that 83 popliteal cysts were removed from 82 patients and communication of the cyst 
with the knee was evident in 54 cases (65\%). In our study, the incidence of communication with the knee joint in the extraarticular ganglion cysts was lower than the reported incidence in Baker's cysts. Of the 7 laterally located extra-articular ganglion cysts in our series, the extra-ganglion cyst was found to be arising from the proximal tibio-fibular joint in 3 cases. In the 23 ganglion cysts, $3 / 23$ cases (13\%) were from the proximal tibio-fibular joint to the extra-articular ganglion cyst. Janzelet al. ${ }^{18)}$ reported that the proximal tibio-fibular joint communicated with the knee joint in $10 \%$ of the patients in their study.

In the current study, an intra-articular cyst extended extraarticularly to an extra-articular cyst in 3 cases (13\%). Although the incidence was very low, the presence of this extension was confirmed with MRI, and thus we suggested intra-articular cyst decompression with open excision for extra-articular ganglion cysts in these patients. Knowledge of the location, characteristic appearance, and distinguishing features of cystic masses around the knee as well as the potential imaging pitfalls, such as atypical cyst contents on MRI, could aid in making definitive diagnoses and proper management plans ${ }^{20)}$. In cases of Baker's cysts, MRI can show the presence of one or more intra-articular lesions in $87 \%-98 \%$ of the cases ${ }^{13,14)}$. Even though there were less intraarticular lesions in our study than those in Baker's cysts (69.6\%), intra-articular pathologies should be carefully taken into consideration in the treatment of extra-articular ganglion cysts around the knee.

In our series, there was only one recurrence after cyst excision. In the patient, 2-3 tiny cysts were observed on follow-up MRI without any symptoms related to the cysts. Two patients had a previous history of simple excision performed at other hospitals and presented to us with recurrence. In these cases, we performed MRI evaluation and found an intra-articular connection of the cyst through the infra-patellar fat pad. Extra-articular procedure (complete cyst excision) with intra-articular procedures such as cyst decompression, meniscectomy, and debridement was performed simultaneously and there was no further recurrence in these cases. Thus, management of a symptomatic extraarticular ganglion cyst around the knee should include thorough MRI evaluation of the knee joint to exclude any associated intraarticular pathology and to know the presence of a communicating channel to the knee joint. Arthroscopic treatment of such lesions and open excision of extra-articular cysts with ligation of the communicating stalk could minimize the chances of recurrence.

Our study has several limitations: the single cohort design without a comparative group; the small sample size $(n=23)$; the relatively short follow-up period (mean, 40 months); and the retrospective design. In addition, the investigation on the presence of a communication channel was limited to the knee joint. Even though the laterally located cysts were connected to the tibio-fibular joint in 3 cases, these cysts were neither connected to the knee joint nor associated with any intra-articular pathology. Another limitation was that we did not take associated intra-articular pathologies, such as meniscal tears and articular cartilage defects, into consideration as the possible cause of pain in patients with a complaint of knee joint pain. However, many of the patients presented with knee discomfort and a palpable mass, and thus we treated the extra-articular cystic mass and intraarticular pathologies with an open and arthroscopic procedure, respectively.

\section{Conclusions}

Arthroscopic management of associated intra-articular pathologies and open cyst excision with ligation of the communicating orifice for the treatment of extra-articular ganglion cysts around the knee joint showed satisfactory clinical outcomes with a low recurrence rate. For adequate treatment of extra-articular ganglion cysts around the knee joint, preoperative MRI evaluation and arthroscopic management of intra-articular pathologies prior to open cyst excision should be considered to diagnose and treat any associated intra-articular pathologies and communications with the knee joint.

\section{Conflict of Interest}

No potential conflict of interest relevant to this article was reported.

\section{References}

1. Bui-Mansfield LT, Youngberg RA. Intraarticular ganglia of the knee: prevalence, presentation, etiology, and management. AJR Am J Roentgenol. 1997;168:123-7.

2. Kang CN, Kim DW, Kim DJ, Kim SJ. Intra-articular ganglion cysts of the knee. Arthroscopy. 1999;15:373-8.

3. Brown MF, Dandy DJ. Intra-articular ganglia in the knee. Arthroscopy. 1990;6:322-3.

4. Kim MG, Kim BH, Choi JA, Lee NJ, Chung KB, Choi YS, Cho SB, Lim HC. Intra-articular ganglion cysts of the knee: clinical and MR imaging features. Eur Radiol. 2001;11:834-40.

5. Butler MG, Fuchigami KD, Chako A. MRI of posterior knee 
masses. Skeletal Radiol. 1996;25:309-17.

6. Narvaez JA, Narvaez J, Aguilera C, De Lama E, Portabella F. MR imaging of synovial tumors and tumor-like lesions. Eur Radiol. 2001;11:2549-60.

7. Valls R, Melloni P, Darnell A, Munoz J, Canalies J. Diagnostic imaging of tibial periosteal ganglion. Eur Radiol. 1997; 7:70-2.

8. Abdelwahab IF, Kenan S, Hermann G, Klein MJ, Lewis MM. Periosteal ganglia: CT and MR imaging features. Radiology. 1993;188:245-8

9. Coakley FV, Finlay DB, Harper WM, Allen MJ. Direct and indirect MRI findings in ganglion cysts of the common peroneal nerve. Clin Radiol. 1995;50:168-9.

10. Kabukcuoglu Y, Kabukcuoglu F, Kuzgun U, Ozturk I. Compression neuropathy of the peroneal nerve caused by a ganglion. Am J Orthop (Belle Mead NJ). 1997;26:700-1.

11. Uetani M, Hashmi R, Hayashi K, Nagatani Y, Narabayashi Y, Imamura K. Peripheral nerve intraneural ganglion cyst: MR findings in three cases. J Comput Assist Tomogr. 1998; 22:629-32

12. Bickel WH, Burleson RJ, Dahlin DC. Popliteal cyst; a clinicopathological survey. J Bone Joint Surg Am. 1956;38:1265-74.

13. Fielding JR, Franklin PD, Kustan J. Popliteal cysts: a reassessment using magnetic resonance imaging. Skeletal Radiol.
1991;20:433-5.

14. Miller TT, Staron RB, Koenigsberg T, Levin TL, Feldman F. MR imaging of Baker cysts: association with internal derangement, effusion, and degenerative arthropathy. Radiology. 1996;201:247-50.

15. Kim JY, Jung SA, Sung MS, Park YH, Kang YK. Extra-articular soft tissue ganglion cyst around the knee: focus on the associated findings. Eur Radiol. 2004;14:106-11.

16. Burk DL Jr, Dalinka MK, Kanal E, Schiebler ML, Cohen EK, Prorok RJ, Gefter WB, Kressel HY. Meniscal and ganglion cysts of the knee: MR evaluation. AJR Am J Roentgenol. 1988;150:331-6.

17. Steiner E, Steinbach LS, Schnarkowski P, Tirman PF, Genant HK. Ganglia and cysts around joints. Radiol Clin North Am. 1996;34:395-425.

18. Janzen DL, Peterfy CG, Forbes JR, Tirman PF, Genant HK. Cystic lesions around the knee joint: MR imaging findings. AJR Am J Roentgenol. 1994;163:155-61.

19. Malghem J, Vande berg BC, Lebon C, Lecouvet FE, Maldague $\mathrm{BE}$. Ganglion cysts of the knee: articular communication revealed by delayed radiography and CT after arthrography. AJR Am J Roentgenol. 1998;170:1579-83.

20. McCarthy CL, McNally EG. The MRI appearance of cystic lesions around the knee. Skeletal Radiol. 2004;33:187-209. 